\title{
Conexão de terminais com limitação de roteadores: complexidade e relação com fluxos e caminhos disjuntos
}

\author{
Alexsander Andrade de Melo ${ }^{1}$ \\ Orientadores: Celina Miraglia Herrera de Figueiredo ${ }^{1}$, Uéverton dos Santos Souza ${ }^{2}$ \\ ${ }^{1}$ COPPE/PESC - Universidade Federal do Rio de Janeiro (UFRJ) \\ Rio de Janeiro - RJ - Brasil \\ ${ }^{2}$ Instituto de Computação - Universidade Federal Fluminense (UFF) \\ Niterói - RJ - Brasil \\ \{aamelo, celina\}@cos.ufrj.br, ueverton@ic.uff.br
}

\begin{abstract}
We investigate the complexity of the TERMINAL CONNECTION PROBLEM (TCP), a network design problem closely related to several classical problems. Particularly, we analyse the strict version of TCP, denoted by S-TCP, when the parameter $r$ is bounded by a constant. We prove that, for $r \in\{0,1\}, S$ TCP is polynomial-time solvable, contrasting with the complexity of TCP, which is known to be NP-complete for all $r \geq 0$. Furthermore, in order to better understand the cases in which $r \geq 2$, we study some variants of the $S$-TCP and relate it to network flow and disjoint path problems. We prove the NPcompleteness of an integral network flow problem, closing a forty-year complexity gap. Finally, we establish a poly vs. NP-c dichotomy for TCP and S-TCP regarding the maximum degree of the input graph and the parameter $\ell$.
\end{abstract}

Resumo. Investigamos a complexidade do PROBLEMA DE CONEXÃO DE TERMINAIS (TCP), um problema de projeto de redes diretamente relacionado a diversos problemas clássicos. Particularmente, analisamos a versão estrita do TCP, denotada por S-TCP, quando o parâmetro r é limitado por uma constante. Provamos que, para $r \in\{0,1\}$, o $S$-TCP é solucionável em tempo polinomial, o que contrasta com a complexidade do TCP, que é sabidamente NP-completo para todo $r \geq 0$. Ademais, com o intuito de compreender melhor os casos em que $r \geq 2$, estudamos algumas variantes do $S$-TCP e o relacionamos com problemas de fluxo em redes e de caminhos disjuntos. Provamos a NP-completude de um problema de fluxo integral, fechando uma questão em aberto por quarenta anos. Por fim, estabelecemos uma dicotomia poly vs. NP-c para o TCP e para o $S$-TCP com respeito ao grau máximo do grafo entrada e ao parâmetro $\ell$.

\section{Introdução}

Problemas de projeto de redes são usualmente questões combinatórias de grande interesse prático e teórico devido ao fato de se relacionarem de maneira próxima a diversas aplicações reais, além de serem de forma geral problemas desafiadores. Neste trabalho, estudamos o problema de projeto de redes denominado PROBLEMA DE CONEXÃO DE TERMINAIS, o qual foi proposto recentemente [Dourado et al. 014a] sob a motivação de aplicações em segurança da informação e em roteamento em redes. 
Dado um grafo $G=(V, E)$ e um conjunto não vazio $W \subseteq V(G)$, dizemos que um subgrafo $T$ de $G$ é uma árvore de conexão para $W$ se as seguintes condições são satisfeitas: (1) $T$ é uma árvore, (2) $W \subseteq V(T)$ e (3) folhas $(T) \subseteq W$, onde folhas $(T)$ denota o conjunto formado pelos vértices que são folhas de $T$. Em uma árvore de conexão $T$ para $W$, os vértices pertencentes a $W$ são chamados de terminais e os vértices pertencentes a $V(T) \backslash W$ são chamados de não terminais, os quais são classificados em duas categorias, de acordo com os seus respectivos graus em $T$, a saber: os vértices não terminais com grau exatamente $2 \mathrm{em} T$ são denominados elos e os com grau ao menos $3 \mathrm{em} T$ são denominados roteadores. Dessa forma, há uma partição $V(T)=W \cup \mathrm{L}(T) \cup \mathrm{R}(T)$ dos vértices da árvore $T$ em terminais, elos e roteadores, onde $\mathrm{L}(T)$ denota o conjunto formado pelos elos de $T$ e $\mathrm{R}(T)$ denota o conjunto formado pelos roteadores de $T$.

Informalmente, o PROBLEMA DE CONEXÃO DE TERMINAIS objetiva conectar um dado conjunto de terminais através de uma árvore de conexão que contenha um número limitado de elos e um número limitado de roteadores. A seguir, apresentamos uma definição formal para o problema.

\begin{tabular}{ll}
\hline PROBLEMA DE CONEX ÃO DE TERMINAIS (TCP) \\
\hline Entrada: & $\begin{array}{l}\text { Um grafo conexo } G, \text { um subconjunto não vazio } W \subseteq V(G) \text { e dois inteiros não } \\
\text { negativos } \ell \text { e } r .\end{array}$ \\
Questão: & O grafo $G$ admite uma árvore de conexão para $W$ tal que $|\mathrm{L}(T)| \leq \ell \mathrm{e}|\mathrm{R}(T)| \leq r ?$ \\
\hline
\end{tabular}

Os autores do problema [Dourado et al. 014a] mostraram que a versão de otimização do TCP pode ser vista como uma generalização de alguns problemas clássicos de projeto de redes, como os problemas da árvore geradora mínima e da árvore de Steiner. Além disso, provaram que o TCP é um problema NP-completo mesmo quando um dos parâmetros: ou $\ell$ ou $r$ é limitado por uma constante; por outro lado, provaram que o TCP é solucionável em tempo polinomial se $\ell$ e $r$ são ambos limitados por constantes.

Por definição, todos os vértices folhas de uma árvore de conexão $T$ pertencem ao conjunto de terminais $W$. Entretanto, note que, não necessariamente todos os vértices terminais são folhas de $T$. Dessa forma, uma árvore de conexão $T$ é dita estrita para um conjunto de terminais $W$ se folhas $(T)=W$. Com base, então, nesse conceito, a versão estrita do TCP, denominada PROBLEMA DE CONEXÃO DE NÓS ESTRITAMENTE TERMINAIS (S-TCP), foi proposta em um trabalho posterior [Dourado et al. 014b], tendo como objetivo determinar se o grafo de entrada $G$ admite uma árvore de conexão estrita para $W$ com no máximo $\ell$ elos e no máximo $r$ roteadores.

De modo análogo ao TCP, o S-TCP foi provado ser NP-completo mesmo quando $\ell$ é limitado por uma constante [Dourado et al. 014b]; como também, foi provado ser solucionável em tempo polinomial se $\ell$ e $r$ são ambos limitados por constantes [Dourado et al. 014b]. No entanto, o caso em que apenas o parâmetro $r$ é limitado por uma constante não havia sido considerado até então.

Assim, com o intuito de preencher essa lacuna, foi estudado durante o mestrado do candidato, de forma central, o S-TCP com $r$ limitado por uma constante. Através desse estudo, provamos que, para $r \in\{0,1\}$, o S-TCP é solucionável em tempo polinomial. No entanto, não conseguimos determinar, até então, a complexidade do problema quando $r$ assume valores constantes maiores ou iguais a 2. Apesar disso, obtivemos resultados parciais que são úteis para uma melhor compreensão do problema. Ademais, consideramos 
variantes do problema onde requer-se que as árvores de conexão satisfaçam propriedades adicionais. Através dessa análise pudemos constatar que o S-TCP está diretamente relacionado a certos problemas de caminhos disjuntos e de fluxo em redes. Desse modo, um segundo objetivo da dissertação de mestrado do candidato é expor essa relação; inclusive, com base no conhecimento adquirido sobre o S-TCP, provamos a NP-completude de um problema de fluxo integral, cuja complexidade permanecia indeterminada por quarenta anos. Por fim, analisamos as complexidades do S-TCP e do TCP quando o grau máximo $\Delta(G)$ do grafo de entrada $G$ é limitado. Mais especificamente, estabelecemos uma dicotomia polinomial vs. NP-completo para o S-TCP e o TCP com relação a $\Delta(G)$ e $\ell$.

Nas seções seguintes descrevemos, de forma resumida, os principais resultados obtidos pelo candidato durante o seu mestrado.

\section{Problema estrito com limitação de roteadores}

Primeiramente, mostramos que, para $r \in\{0,1\}$, o S-TCP é solucionável em tempo polinomial, conforme enunciamos no teorema a seguir, o que constrasta com a complexidade do TCP que é NP-completo para todo $r \geq 0$.

Teorema 2.1. Para $r \in\{0,1\}$, o S-TCP é solucionável em tempo polinomial.

O algoritmo que propomos para o S-TCP com $r=0$ consiste, simplesmente, em uma análise da cardinalidade do conjunto de terminais $W$ e em determinar a distância em $G$ entre os dois possíveis terminais pertencentes a $W$. Por outro lado, o algoritmo que propomos para o S-TCP com $r=1$ consiste em uma redução de Turing para o problema de caminhos disjuntos denominado MIN-SUM st-CAMINHOS DISJUNTOS, cujo objetivo é determinar se um dado grafo $G^{\prime}$ contém $k$ caminhos internamente disjuntos em vértices tais que: os seus extremos são um dado par de vértices $\{s, t\}$ e a soma de seus comprimentos é no máximo $x$, onde $k$ e $x$ são inteiros não negativos dados na entrada do problema. Esses resultados foram apresentados no VII Latin American Workshop on Cliques in Graphs e foram aceitos para publicação na revista Matemática contemporânea.

\subsection{Resultados parciais para o caso $r \geq 2$}

Embora desconheçamos, até então, a complexidade do S-TCP para valores de $r$ constantes maiores ou igual 2, obtivemos ao longo de nossos estudos resultados parciais que são de grande utilidade para uma melhor compreensão do problema. As proposições seguintes exemplificam esses resultados.

Proposição 2.1. Para $r$ constante e $r \geq|R|$, existe uma redução de Turing do S-TCP para a versão do problema, denotada por $\mathrm{S}-\mathrm{TCP}(R)$, onde requer-se que o conjunto de roteadores da árvore de conexão estrita seja precisamente um dado conjunto $R$.

Proposição 2.2. A versão relaxada do S-TCP na qual não se limita a quantidade de elos é solucionável em tempo polinomial se $r$ e $|W|$ são limitados por constantes.

Proposição 2.3. Para todo $c \geq 0$, existe uma redução polinomial do S-TCP $\operatorname{com} r=c$ para o S-TCP com $r=c+1$.

\subsection{Restrições adicionais}

Ainda com o objetivo de obtermos mais intuição sobre o S-TCP, especialmente quando $r$ assume valores constantes maiores ou iguais a 2, investigamos a complexidade de algumas variantes do S-TCP que contêm restrições adicionais, sobre as quais discorremos agora. 
A primeira variante que consideramos é denominada SUBGRAFO DE ROTEADORES CONEXO S-TCP, e tem como objetivo determinar se $G$ admite uma árvore de conexão estrita $T$ para $W$ com no máximo $\ell$ elos e $r$ roteadores tal que o subgrafo de $T$ induzido por $\mathrm{R}(T)$ é conexo. Essa variante se trata, na verdade, de um problema NPcompleto (veja Teorema 3.16 da dissertação), no entanto provamos, novamente através de uma redução de Turing para o MIN-SUM st-CAMINHOS DISJUNTOS, que se $r$ é limitado por uma constante, então a mesma pode ser solucionada em tempo polinomial.

Teorema 2.2. Para todo $r \geq 1$ constante, SubGRAFO DE ROTEAdORES CONEXO STCP é solucionável em tempo polinomial.

Corolário 2.1. Para $|R|=2$, o $\mathrm{S}-\mathrm{TCP}(R)$ é solucionável em tempo polinomial se o subgrafo $G[R]$ é conexo.

Outra variante do S-TCP que investigamos é denominada CONJUNTO DE TERMINAIS PARTICIONADO S-TCP, a qual, adicionalmente, recebe em sua entrada uma partição $W=W_{1} \cup W_{2} \cup \cdots \cup W_{r^{\prime}}$, onde $r^{\prime} \leq r$, e tem como objetivo determinar se $G$ admite uma árvore de conexão estrita $T$ para $W$ com no máximo $\ell$ elos e $r$ roteadores tal que: para todo $i \in\left\{1,2, \ldots, r^{\prime}\right\}$, os terminais em $W_{i}$ são conectados a $T$ através de um mesmo roteador, i.e. existe $\rho \in \mathrm{R}(T)$ tal que, para todo $w \in W_{i}$, $\operatorname{dist}_{T}(w, \rho)=\operatorname{dist}_{T}(w, \mathrm{R}(T))$.

Teorema 2.3. Para todo $r \geq 2$, CONJUNTO DE TERMINAIS PARTICIONADO S-TCP é NP-completo.

Por fim, analisamos a variante do S-TCP denominada SEMIESTRUTURAdo STCP, a qual, adicionalmente, recebe em sua entrada um subconjunto $R \subseteq V(G) \backslash W$, tal que $|R| \leq r$, e uma topologia de conexão $T_{R}$ para os vértices pertencentes a $R$; e tem como objetivo determinar se $G$ admite uma árvore de conexão estrita $T$ para $W$ com no máximo $\ell$ elos e $r$ roteadores tal que: $\mathrm{R}(T) \supseteq R$ e, para toda aresta $\rho_{i} \rho_{j} \in E\left(T_{R}\right)$, o caminho em $T$ entre $\rho_{i}$ e $\rho_{j}$ não contém quaisquer outros roteadores.

Teorema 2.4. Para todo $|R| \geq 2$ e $r \geq 3$, tais que $r \geq|R|$, Semiestruturado S-TCP é NP-completo.

\subsection{Complexidade parameterizada}

Dourado et al. [Dourado et al. 014b] investigaram a complexidade do S-TCP quando $\Delta(G), \ell, r$ são parâmetros fixos; mais especificamente, provaram que esta versão parameterizada do problema é FPT. No lado oposto, provamos que, se $\Delta(G)$ não é tido como parâmetro fixo, então é improvável a existência de um algoritmo FPT para o S-TCP parametrizado por $\ell, r$. Na verdade, de maneira mais restritiva, provamos que o S-TCP parametrizado por $r$ é W[2]-difícil mesmo quando $\ell$ é limitado por uma constante.

Teorema 2.5. Para todo $\ell \geq 0$, o S-TCP parametrizado por $r$ é W[2]-difícil.

Este último resultado foi provado através de uma redução FPT para o problema Cobertura POR CONJuntos, que é um problema W[2]-difícil clássico. Como uma consequência adicional desta redução, temos que o TCP parametrizado por $r$ é também $\mathrm{W}[2]$-difícil mesmo quando $\ell$ é limitado por uma constante.

Corolário 2.2. Para todo $\ell \geq 0$, o TCP parametrizado por $r$ é W[2]-difícil.

\section{Fluxo integral de dois commodities e $k+1$ caminhos disjuntos}

Com base nos resultados apresentados, pode-se verificar que o S-TCP com $r=2$ é polinomialmente redutível ao problema denominado $k+1$ CAMINHOS DISJUNTOS EM VÉR- 
TICES, cujo objetivo é responder se um grafo dado admite $k+1$ caminhos disjuntos em vértices tais que: $k$ caminhos são entre um dado par de vértices e um caminho é entre um segundo dado par de vértices, quando exige-se que o somatório dos comprimentos desses caminhos seja limitado. Um problema relacionado ao $k+1$ CAMINHOS DISJUNTOS EM VÉRTICES é o $k+1$ CAMINHOS DISJUNTOS EM ARESTAS, versão onde permite-se que os caminhos compartilhem vértices mas não arestas. Este segundo problema pode ser interpretado como um problema de fluxo integral de dois commodities em grafos não direcionados, onde a capacidade das arestas e a demanda de um dos commodities é unitária.

Na literatura há uma ampla coletânea de resultados sobre problemas de caminhos disjuntos e fluxo em redes [Karp 1975, Even et al. 1976, Robertson and Seymour 1995]. Entretanto os casos particulares descritos no parágrafo anterior, curiosamente, não haviam sidos considerados até então. Por exemplo, a complexidade do problema de fluxo em redes SIMPLE U2CIF quando a demanda de um dos commodities é unitária, mencionado acima, permanecia indeterminada por quarenta anos [Even et al. 1976]. Dessa forma, investigamos a complexidade de tais problemas, e provamos os teoremas enunciados abaixo, os quais foram apresentados no IX Latin and American Algorithms, Graphs and Optimization Symposium, com publicação Eletronic Notes in Discrete Mathematics [Melo et al. 2017].

Teorema 3.1. O problema SIMPLE U2CIF é NP-completo mesmo se a demanda de um dos seus dois commodities é unitária.

Teorema 3.2. O problema $k+1$ CAMINHOS DISJUNTOS EM VÉRTICES é NP-completo.

\section{Grau máximo limitado}

Nesta seção, analisamos tanto a complexidade do TCP quanto a complexidade do S-TCP quando o grau máximo $\Delta(G)$ do grafo de entrada $G$ é limitado.

Observamos que, nas demonstrações do Teorema 2.1 [Dourado et al. 014a] e do Corolário [Dourado et al. 014b], onde as NP-completudes do TCP e do S-TCP, com $\ell$ limitado por uma constante, são respectivamente estabelecidas, é construído um grafo $G$ cujo grau máximo é arbitrariamente grande. No entanto, no Teorema 7 [Dourado et al. 014b] é apresentado uma forma de modificar essas demonstrações de modo que seja, então, possível concluir que o TCP e o S-TCP, com $\ell$ limitado por uma constante, permanecem NP-completos mesmo quando restritos aos grafos com grau máximo limitado. Apesar disso, a constante que limita o grau máximo do grafo $G$ construído é consideravelmente grande, contudo, e, assim, não suficiente para se estabelecer uma fronteira que determine quando o TCP ou o S-TCP admitem algoritmo de tempo polinomial ou quando tais problemas permanecem NP-completos, em função de $\Delta(G)$.

Assim, sob a motivação de estabelecer tal fronteira, provamos os resultados enunciados nos teoremas abaixo, os quais, juntamente com a observação de que o TCP e o S-TCP são facilmente solucionáveis em tempo polinomial quando $\Delta(G) \leq 2$, definem uma dicotomia polinomial versus NP-completo para o TCP e o S-TCP no que diz respeito aos parâmetros $\Delta(G)$ e $\ell$.

Teorema 4.1. Para todo $\ell \geq 0$, o TCP é NP-completo mesmo se $\Delta(G)=3$.

Teorema 4.2. Para todo $\ell \geq 0$, o S-TCP é NP-completo mesmo se $\Delta(G)=4$.

Teorema 4.3. O S-TCP é NP-completo mesmo se $\Delta(G)=3$. 
Teorema 4.4. Para todo $\ell \geq 0$ constante, o S-TCP é solucionável em tempo polinomial quando $\Delta(G)=3$.

Adicionalmente, analisamos o TCP quando $\Delta(G)$ e $r$ são limitados por constantes, e, conforme enunciado no teorema a seguir, provamos que, para todo $r \geq 0$, o TCP permanece NP-completo mesmo se $\Delta(G)=3$, definindo também, assim, uma dicotomia polinomial versus $N P$-completo para o TCP com respeito aos parâmetros $\Delta(G)$ e $r$.

Teorema 4.5. Para todo $r \geq 0$, o TCP é NP-completo mesmo se $\Delta=3$.

\section{Conclusão}

Neste trabalho, apresentamos contribuições importantes para os problemas de projeto de redes TCP e S-TCP. Especialmente, analisamos o S-TCP quando o parâmetro $r$ é limitado por uma constante. Além disso, estudamos diversas variantes do problema e mostramos que o S-TCP está diretamente ligado a problemas de fluxos em redes e caminhos disjuntos. Adicionalmente, investigamos a complexidade do TCP e do S-TCP quando $\Delta(G)$ e $\ell$ são limitados por constantes, e do TCP quando $\Delta(G)$ e $r$ são limitados por constantes.

Além das publicações mencionadas ao longo do texto, uma parte dos resultados obtidos foi submetida para o special issue do IX Latin and American Algorithms, Graphs and Optimization Symposium, que será publicado na revista Discrete Applied Mathematics, e outra parte será submetida para o Journal of Computer and System Sciences.

\section{Referências}

Dourado, M. C., Oliveira, R. A., Protti, F., and Souza, U. S. (2014a). Design of connection networks with bounded number of non-terminal vertices. In proceedings of $V$ LatinAmerican Workshop on Cliques in Graphs, LAWCG 2012, volume 42 of Matemática Contemporânea, pages 39-47. SBM.

Dourado, M. C., Oliveira, R. A., Protti, F., and Souza, U. S. (2014b). Conexão de terminais com número restrito de roteadores e elos. In annals of XLVI Simpósio brasileiro de pesquisa operacional, pages 2965-2976.

Even, S., Itai, A., and Shamir, A. (1976). On the complexity of timetable and multicommodity flow problems. SIAM Journal on Computing, 5(4):691-703.

Karp, R. M. (1975). On the computational complexity of combinatorial problems. Networks, 5:45-68.

Melo, A. A., de Figueiredo, C. M. H., and Souza, U. S. The strict terminal connection problem with a bounded number of routers. In proceedings of VII Latin-American Workshop on Cliques in Graphs, LAWCG 2016, Matemática Contemporânea (to appear).

Melo, A. A., de Figueiredo, C. M. H., and Souza, U. S. (2017). Simple undirected twocommodity integral flow with a unitary demand. In proceedings of Latin and American Algorithms, Graphs and Optimization Symposium, LAGOS 2017, volume 62 of Eletronic Notes in Discrete Mathematics, pages 279-284.

Robertson, N. and Seymour, P. D. (1995). Graph minors. xiii. the disjoint paths problem. Journal of Combinatorial Theory, Series B, 63(1):65-110. 